\title{
SONOLÊNCIA E FADIGA EM TRABALHADORES DIURNOS E NOTURNOS ${ }^{1}$
}

\author{
Sleepiness and fatigue among day and night workers
}

LUI REINHARDT, Érica ${ }^{2}$

FISCHER, Frida Marina ${ }^{3}$

\begin{abstract}
REsumo
A sonolência corresponde à maior propensão a adormecer e eleva-se com o trabalho noturno. A fadiga é uma sensação de tensão ou exaustão induzida por esforço e aumenta com turnos diurnos ou noturnos. O objetivo foi avaliar duração do sono, sonolência, fadiga e outras variáveis entre trabalhadores diurnos e noturnos. Os dados foram obtidos por questionários e actimetria. Os grupos não diferiram quanto à duração do sono, mas os trabalhadores noturnos relataram pior qualidade $e$ aumento significativo da sonolência. Todos relataram aumento significativo da fadiga, mais pronunciado entre trabalhadores noturnos. Esses resultados confirmam a associação do trabalho noturno com prejuízos ao sono, sonolência e fadiga. Más condições de trabalho associaram-se a níveis mais elevados de estresse, maior sonolência e fadiga. Avaliações em segurança e saúde no trabalho devem também abranger a sonolência e a fadiga dos trabalhadores e fatores associados.
\end{abstract}

Palavras-chave: Sonolência. Fadiga. Jornada de trabalho em turnos.

\section{ABSTRACT}

Sleepiness means a heightened propensity to fall asleep that increases with night work. Fatigue is a feeling of strain or exhaustion induced by effort that increases with day or night work shift. The aim of this study was to evaluate sleep duration, perceived sleepiness and fatigue, and other variables among permanent day and night workers. The data were obtained by questionnaires and actimetry. Workers didn't differ significantly regarding sleep duration, but night workers perceived worse sleep quality and a significant increase in sleepiness. Increase of fatigue was significant for all workers, more pronounced for night workers. These results confirm the association of night work with disturbed sleep, sleepiness and fatigue. Poor working conditions were associated with greater perceived stress, sleepiness and fatigue. Evaluations of occupational safety and health should also cover workers sleepiness and fatigue and associated variables.

Keywords: Sleepiness. Fatigue. Shift work schedule.

\footnotetext{
${ }^{1}$ Este texto descreve e analisa parte dos resultados obtidos durante pesquisa de doutorado realizada na Faculdade de Saúde Pública da USP, previamente aprovada pelo respectivo Comitê de Ética em Pesquisa, processo CAAE 0105.0.207.000-09. A pesquisa recebeu financiamento da Fundacentro, da FAPESP (processo 2011/10503-4) e da CAPES (auxílio 8755-11/6).

${ }^{2}$ Doutora em Saúde Pública, Mestre em Biotecnologia e Especialista em Saúde Pública pela USP. Graduação em Ciências Biológicas pela USP. Pesquisadora da Fundacentro em São Paulo, SP. E-mail: erica.reinhardt@fundacentro.gov.br.

${ }^{3}$ Pós-doutora pelo Arbeitsphysiologie Institut em Dortmund, Alemanha, Doutora e Mestre em Saúde Pública pela USP e Especialista em Ergonomia pela USP. Professora titular da Faculdade de Saúde Pública da Universidade de São Paulo. E-mail: fmfische@usp.br.
}

Trabalho \& Educação | v.30 | n.1 | p.53-67 | jan-abr | 2021 


\section{INTRODUÇÃO}

Sonolência e fadiga são fenômenos relacionados e por vezes tratados como equivalentes, mas há boas razões para diferenciá-los (AKERSTEDT; WRIGHT, 2009; PHILLIPS, 2015; SHEN; BARBERA; SHAPIRO, 2006).

A sonolência é extremamente comum, multidimensional e multicausal (SHEN; BARBERA; SHAPIRO, 2006). Operacionalmente seria definida como a tendência ou propensão para dormir (AKERSTEDT; WRIGHT, 2009; SHEN; BARBERA; SHAPIRO, 2006) causada por alteração ou desequilíbrio nos mecanismos associados ao sono ou à vigília (SHEN; BARBERA; SHAPIRO, 2006). Estes derivam de atividades neuronais intrínsecas no sistema nervoso central, sob influência dos ritmos biológicos, e de vários fatores homeostáticos, ambientais e comportamentais (SHEN; BARBERA; SHAPIRO, 2006). É considerada excessiva se aumentada em circunstâncias indesejadas ou inapropriadas (BERTOLAZI et al., 2009; SHEN; BARBERA; SHAPIRO, 2006) e frequentemente decorre de perturbações no sono ou dessincronização circadiana ocasionadas pelo trabalho (AKERSTEDT; WRIGHT, 2009).

No entanto, os mecanismos relacionados ao sono e à vigília não estão obrigatoriamente implicados na fadiga, como ocorre com a sonolência (SHEN; BARBERA; SHAPIRO, 2006).

A fadiga pode ser definida como uma condição psicofisiológica subótima causada por algum tipo de esforço (PHILLIPS, 2015) e que corresponde subjetivamente a uma sensação de tensão ou de exaustão (SHAHID; SHEN; SHAPIRO, 2010). Ela pode ser causada por um esforço exercido ou ainda em exercício e sob a interferência de ampla gama de fatores, incluindo os rítmicos e circadianos, privação de sono, falta de períodos de descanso, temperaturas extremas, má iluminação, pressão de tempo e sobrecarga de trabalho e fatores externos ao trabalho (PHILLIPS, 2015).

A fadiga fisiológica ou aguda é uma função protetiva normal do organismo que geralmente ocorre em indivíduos saudáveis (SHEN; BARBERA; SHAPIRO, 2006). Ela tem um início rápido e curta duração, usualmente deve-se a uma única causa, pode ser aliviada após um período de recuperação sem dormir (AKERSTEDT; WRIGHT, 2009; SHAHID; SHEN; SHAPIRO, 2010; SHEN; BARBERA; SHAPIRO, 2006) e geralmente exerce efeitos muito pequenos nas atividades diárias ou na qualidade de vida (SHEN; BARBERA; SHAPIRO, 2006). Uma distinção prática útil entre a fadiga e a sonolência é que esta última é exacerbada por um período de inatividade ou descanso, efeito contrário ao relativo à fadiga (AKERSTEDT; WRIGHT, 2009).

Por outro lado, situações de esforço continuado ou repetido levam à fadiga crônica, que podem incluir estados de exaustão sem sonolência que se associam ao estresse e ao burnout (PHILLIPS, 2015). Ela afeta principalmente populações com desordens clínicas e é percebida como anormal ou excessiva (SHEN; BARBERA; SHAPIRO, 2006). Geralmente tem início insidioso, é persistente e de etiologia multifatorial, não sendo aliviada com as técnicas restauradoras usuais e afetando as atividades diárias e a qualidade de vida de forma significativa (SHEN; BARBERA; SHAPIRO, 2006).

O impacto da sonolência e da fadiga no trabalho são semelhantes, acarretando diminuição de produtividade, aumento de absenteísmo, maiores índices de acidentes e uma maior probabilidade de incapacitação ocasionada por doença relacionada (AKERSTEDT; WRIGHT, 2009; BERTOLAZI et al., 2009; SHEN; BARBERA; SHAPIRO, 2006). 
Sonolência e fadiga estão associadas ao trabalho em turnos, o que inclui o trabalho noturno. O trabalho em horários não usuais é obrigatório apenas quando o processo produtivo ou o serviço à população não podem ser interrompidos, sob pena de prejuízos materiais ou sociais importantes. $\mathrm{O}$ ideal, portanto, seria que somente trabalhadores dessas atividades estivessem submetidos a esse esquema de trabalho. Na realidade, em muitos casos observa-se que a adoção do trabalho em turnos obedece exclusivamente à lógica de mercado e visa atender sobretudo aos interesses e à conveniência das empresas e do mercado consumidor, configurando-se em autêntica precarização do trabalho (ANTUNES, 2009; FISCHER, 2004).

Por isso, considerando o potencial de sonolência e fadiga afetarem negativamente a saúde e o bem-estar de trabalhadores, com efeitos negativos também para o trabalho, foi desenvolvida esta pesquisa com trabalhadores diurnos e noturnos com o objetivo de contribuir para a compreensão desses fenômenos e fatores associados.

\section{MATERIAL E MÉTODOS}

Esta foi uma pesquisa de desenho transversal em empresa da cidade de São Paulo que fabrica principalmente metais sanitários, comparando trabalhadores noturnos (turno das $21 \mathrm{~h}$ às $6 \mathrm{~h}$ ) com diurnos (turno das $7 \mathrm{~h}$ às $17 \mathrm{~h}$ ). $\mathrm{O}$ ciclo de turnos estendia-se por 7 dias, compreendendo 5 turnos fixos, diurnos ou noturnos, iniciados às segundas-feiras, e 2 dias de folga, abrangendo o sábado e o domingo, quando então a empresa permanecia fechada. Além disso, nem todos os setores operavam 24 horas por dia de segunda a sexta-feira, incluindo alguns dos setores operacionais. Assim, a empresa adotava esquema de turnos semicontínuos (FISCHER, 2004).

Participaram dezenove trabalhadores do sexo masculino do turno diurno e dezesseis do turno noturno, com idades entre 20 e 45 anos e pelo menos 4 meses de trabalho nos turnos selecionados. Os trabalhadores noturnos foram divididos em dois subgrupos, conforme descrito adiante.

Todos os trabalhadores noturnos optaram por este turno devido ao adicional noturno. Entre os trabalhadores diurnos a situação inverte-se: quatorze não optaram por este turno diurno, sendo que cinco informaram ter iniciado neste turno de trabalho a partir de demanda da empresa. No entanto, mesmo nestes casos, muitos deles afirmaram ter preferência pelo turno diurno.

Foram excluídos trabalhadores que relataram diagnóstico de problema de saúde com possível interferência sobre as variáveis do estudo, além daqueles que faziam uso de medicamentos relacionados ou abuso de álcool ou drogas nos doze meses anteriores ao estudo.

Dados sociodemográficos, de saúde e de condições de vida e trabalho foram obtidos por questionário adaptado de Borges (BORGES, 2006). A sonolência foi inicialmente caracterizada com o uso da escala de sonolência de Epworth (BERTOLAZl et al., 2009; PIRES et al., 2007), com o máximo de 24 pontos, e a fadiga por meio de questionário relacionado ao trabalho (YOSHITAKE, 1971), de 0 a 120 pontos. Também foi avaliado o estresse percebido (REIS; HINO; AÑNEZ, 2010), com escore global máximo de 40 pontos.

Para avaliar o sono, os trabalhadores preencheram um protocolo de atividades diárias (FISCHER, 1985) por dez dias consecutivos, iniciando numa sexta-feira, com o uso concomitante de actímetros (AMBULATORY MONITORING INC., 2000), permitindo 
determinar variáveis relativas ao sono com maior exatidão. O sono do quinto dia de trabalho tanto diurno quanto noturno (ou seja, o sono noturno de sexta-feira dos trabalhadores diurnos e o sono diurno de sábado dos trabalhadores noturnos) foi excluído das análises. Também foi excluído o sono do segundo dia de folga (sono de domingo para segunda-feira para todos os trabalhadores). Os motivos dessas exclusões é que esses períodos de sonos sofrem mudanças abruptas devido à transição entre dias de trabalho e de folga, o que faz com que divirjam quali e quantitativamente tanto dos sonos dos dias de trabalho quanto dos dias de folga.

Os trabalhadores também preencheram a Escala de Sonolência de Karolinska (Karolinska Sleepiness Scale - KSS), uma escala com nove pontos (AKERSTEDT; GILLBERG, 1981), e uma escala visual analógica de $10 \mathrm{~cm}$ para avaliação da fadiga em três momentos do turno de trabalho: no início, aproximadamente no meio (entre $13 \mathrm{~h} 00$ e 14h00 para os trabalhadores diurnos, e às $3 \mathrm{~h} 00$ para os trabalhadores noturnos) e no final do turno. Para as análises foram consideradas as médias dos resultados dos primeiros quatro dias de trabalho, pois o último dia de trabalho do ciclo de turnos tem características diferenciadas que poderiam interferir nessas variáveis subjetivas.

Todos os resultados foram submetidos a análises estatísticas no software IBM SPSS 20 (IBM, Inc., Estados Unidos da América). A normalidade na distribuição das variáveis foi analisada em histogramas e por testes de Shapiro-Wilk e determinou o uso dos testes de hipóteses paramétricos ou não-paramétricos subsequentes. Foram considerados como diferenças significativas valores de p inferiores a 0,05.

Este estudo atendeu ao requerido na Declaração de Helsinki e foi aprovado por Comitê de Ética em Pesquisa formalmente constituído (processo CAAE 0105.0.207.000-09).

\section{Resultados}

\section{Caracterização da população e procedimentos iniciais}

Trabalhadores diurnos e noturnos não exerciam as mesmas funções e pertenciam a diferentes setores da empresa. Os trabalhadores noturnos distribuíam-se entre os setores de macharia, policorte e rebarbação de peças e fundição de metais, todos no mesmo espaço físico da fundição, e o setor de afinação. Verificou-se uma grande exigência física nas atividades de fundição de metais, incluindo cinco coquilheiros avaliados neste estudo. A maior exigência deve-se ao manuseio dos moldes metálicos (coquilhas) bastante pesados, com cerca de $5 \mathrm{~kg}$, e à sua grande exposição ao calor, oriundo dos fornos de fundição de metais, com temperaturas nas faixas entre 960 a $1050^{\circ} \mathrm{C}$, e do próprio metal em estado líquido vazado no interior das coquilhas mantidas unidas pelos coquilheiros. Estando no mesmo espaço físico da fundição, outros três trabalhadores com as funções de rebarbador, macheiro e auxiliar de produção também estavam expostos ao calor excessivo oriundo dos fornos e processos produtivos da fundição de metais. Constatou-se também que esses oito trabalhadores, exceto um, desenvolviam suas atividades em pé durante toda a noite e ainda estavam expostos aos fumos metálicos característicos da fundição de metais, aumentando seu risco de intoxicação e contribuindo para um mau cheiro constante neste ambiente, tornando-o mais perigoso e desagradável que o ambiente dos outros trabalhadores avaliados neste estudo, diurnos ou noturnos. 
Dessa forma, esses oito trabalhadores, todos do turno noturno, puderam ser reunidos em um subgrupo com maior desgaste ocupacional, com reflexo nas variáveis de interesse deste estudo. Eles constituíram o subgrupo de trabalhadores noturnos da fundição. Os oito trabalhadores noturnos restantes trabalhavam como afinadores no setor de afinação, constituindo o subgrupo dos trabalhadores noturnos da afinação. Embora as atividades da afinação não envolvessem manusear pesos e não fosse produzido calor excessivo ou fumos metálicos, ainda assim esses afinadores passavam todo o turno em pé, em uma única tarefa específica: segurando e girando a peça fundida com as duas mãos e encostando toda sua superfície em uma lixa em movimento para obter todo o primeiro acabamento. Nessa atividade havia risco constante de acidente no contato da lixa com a mão, razão por que vários trabalhadores enrolavam fita crepe nos dedos.

Já os trabalhadores diurnos distribuíam-se em dez setores diferentes, inviabilizando subdivisões para análises estatísticas posteriores. Por isso, e considerando que as atividades e condições de trabalho eram semelhantes, esses trabalhadores foram tratados como um grupo único, sem nenhuma subdivisão.

No geral, nas funções e tarefas desempenhadas em ambos os turnos observou-se o predomínio de tarefas relativamente simples, repetitivas e com baixa autonomia do trabalhador. Além disso, as funções e tarefas nos dois turnos possuíam exigências físicas e mentais semelhantes, à exceção daquelas do setor de fundição, com atividades com maior exigência física e piores condições ambientais.

Os trabalhadores tinham 31,7 $\pm 6,7$ anos de idade e uma mediana de 3,5 anos de trabalho no turno específico. Os três grupos não diferiram significativamente em relação a essas variáveis e às demais variáveis sociodemográficas, de saúde e condições de vida. Tratava-se de uma população jovem e sem doenças crônicas ou outros problemas de saúde, cuja escolaridade não ia além do ensino médio e trabalhando em ocupações braçais, subalternas, desgastantes e de baixa remuneração.

Os grupos também não apresentaram diferenças estatisticamente significativas em relação às escalas de sonolência de Epworth e de fadiga relacionada ao trabalho. A mediana do escore da sonolência foi de 7 pontos, com intervalo interquartil (IQ) de 3 a 10 pontos, enquanto a fadiga média foi de $24,49 \pm 15,35$ pontos. Nos dois casos a pontuação ficou bem abaixo da pontuação média de cada escala, sugerindo que esses trabalhadores não estavam cronicamente sonolentos ou cansados.

Os grupos diferiram quanto ao estresse percebido, em que os trabalhadores noturnos da afinação apresentaram resultados melhores quando comparados aos outros grupos, que não diferiram entre si. O estresse percebido dos trabalhadores noturnos da afinação (mediana $=3,5 ; \mathrm{IQ}=0$ a 5,5) foi significativamente menor, pelo teste post hoc de Dunn, àquele dos trabalhadores noturnos da fundição (mediana $=14 ; \mathrm{IQ}=8,75$ a 19,50; $p<$ 0,007 , com correção de Bonferroni) e àquele dos trabalhadores diurnos (mediana = 13; $\mathrm{IQ}=9$ a 17; $p<0,003$, com correção de Bonferroni). Apenas três trabalhadores diurnos e um trabalhador noturno da fundição registraram estresse percebido acima de 20 pontos, correspondente ao valor intermediário da escala.

\section{Análise do sono}

Os resultados da análise do sono dos três grupos de trabalhadores encontram-se na Tabela 1. 
Em média, os trabalhadores noturnos passaram quase uma hora a mais na cama se considerado o sono total em dias de trabalho. A duração do sono total também tendeu a ser maior entre os trabalhadores noturnos. Mas essas diferenças resultaram não significativas (Tabela 1).

No dia de folga todos os trabalhadores diurnos e noturnos tiveram o sono principal na noite de sábado para domingo. Nesse dia, apenas um dos trabalhadores noturnos da fundição teve mais de um episódio de sono, tendo dormido muito menos que os outros trabalhadores noturnos da fundição. Por essas razões ele foi considerado um outliere foi excluído das análises relativas ao sono do dia de folga. O tempo passado na cama e a duração do sono foram pelo menos duas horas mais longas entre os trabalhadores noturnos que entre os diurnos (Tabela 1).

Após testes post hoc de Games-Howell, resultaram significativas as diferenças entre trabalhadores diurnos e noturnos da afinação em relação ao tempo na cama $(p<0,013)$ e à duração do sono $(p<0,046)$. Também foram significativas as diferenças de tempo na cama e da duração de sono dos trabalhadores diurnos e dos noturnos da fundição ( $p$ $<0,023$ e $p<0,031$, respectivamente). Não houve diferenças estatisticamente significativas entre os trabalhadores noturnos da afinação e da fundição.

Tabela 1. Resultados relativos ao sono dos trabalhadores diurnos ( $N=19)$, noturnos da afinação ( $N$ =8) e noturnos da fundição ( $N=8)$.

\begin{tabular}{|c|c|c|c|c|}
\hline Variável & Grupo & $\begin{array}{c}\text { Resultados da } \\
\text { variável }\end{array}$ & $\begin{array}{c}\text { Resultados da } \\
\text { ANOVA de Welch }\end{array}$ & $\begin{array}{c}\text { Significância } \\
\text { (bicaudal) }\end{array}$ \\
\hline Tempo médio na cama & Diurno & $6: 03 \pm 1: 01$ & & \\
\hline \multirow{2}{*}{$\begin{array}{l}\text { em dias de trabalho - } \\
\text { sono principal(1) }\end{array}$} & Afinação & $6: 29 \pm 1: 02$ & $F(2 ; 32)=0,456$ & $p=0,643$ \\
\hline & Fundição & $6: 10 \pm 1: 15$ & & \\
\hline Tempo médio na cama & Diurno & $6: 03 \pm 1: 01$ & & \\
\hline \multirow{2}{*}{$\begin{array}{l}\text { em dias de trabalho - } \\
\text { sono total(2) }\end{array}$} & Afinação & $6: 51 \pm 0: 52$ & $F(2 ; 32)=2,925$ & $p=0,084$ \\
\hline & Fundição & $6: 55 \pm 1: 04$ & & \\
\hline \multirow{3}{*}{$\begin{array}{l}\text { Duração média em dias } \\
\text { de trabalho - sono } \\
\text { principal }\end{array}$} & Diurno & $4: 44 \pm 1: 06$ & & \\
\hline & Afinação & $5: 13 \pm 1: 29$ & $F(2 ; 32)=0,386$ & $p=0,687$ \\
\hline & Fundição & $5: 00 \pm 1: 29$ & & \\
\hline \multirow{3}{*}{$\begin{array}{l}\text { Duração média em dias } \\
\text { de trabalho - sono total }\end{array}$} & Diurno & $4: 44 \pm 1: 06$ & & \\
\hline & Afinação & $5: 32 \pm 1: 29$ & $F(2 ; 32)=1,393$ & $p=0,283$ \\
\hline & Fundição & $5: 25 \pm 1: 20$ & & \\
\hline \multirow{3}{*}{$\begin{array}{l}\text { Tempo na cama no dia } \\
\text { de folga }\end{array}$} & Diurno & $7: 24 \pm 1: 11$ & & \\
\hline & Afinação & $9: 22 \pm 1: 24$ & $F(2 ; 31)=10,167$ & $p<0,004$ \\
\hline & Fundição(3) & $10: 36 \pm 2: 17$ & & \\
\hline \multirow{3}{*}{$\begin{array}{l}\text { Duração do sono no dia } \\
\text { de folga }\end{array}$} & Diurno & $5: 34 \pm 1: 53$ & & \\
\hline & Afinação & $7: 33 \pm 1: 43$ & $F(2 ; 31)=6,632$ & $p<0,012$ \\
\hline & Fundição(3) & $8: 59 \pm 2: 37$ & & \\
\hline
\end{tabular}

(1) sono principal: maior episódio de sono em 24 horas; (2) sono total: todos episódios de sono registrados em 24 horas, inclusive cochilos; (3) $\mathrm{N}=7$ após a exclusão do outlier 
Testes $t$ pareados confirmaram que o sono do primeiro dia de folga (sábado para domingo) foi significativamente mais longo para todos os trabalhadores comparado àquele de dias de trabalho, correspondendo ao rebote de sono. Os trabalhadores noturnos dormiram significativamente mais tempo nesse dia que os diurnos, sugerindo que o turno noturno potencializou esse rebote no dia de folga.

\section{Análise da sonolência e da fadiga}

Inicialmente, os resultados de sonolência e fadiga foram comparados entre os três grupos de trabalhadores pelo teste de Kruskal-Wallis. Conforme a Tabela 2, nenhuma das comparações foi significativa.

Tabela 2. Sonolência e fadiga durante o turno de trabalho de trabalhadores diurnos $(\mathrm{N}=19)$, noturnos da afinação $(\mathrm{N}=8)$ e noturnos da fundição $(\mathrm{N}=8)$.

\begin{tabular}{|c|c|c|c|c|}
\hline Variável & Grupo & Resultados da variável(1) & $\begin{array}{c}\text { Resultados do } \\
\text { teste de Kruskal- } \\
\text { Wallis }\end{array}$ & $\begin{array}{c}\text { Significância } \\
\text { (bicaudal) }\end{array}$ \\
\hline \multirow{3}{*}{$\begin{array}{l}\text { Sonolência média no } \\
\text { início do turno }\end{array}$} & Diurno & 3,$00 ; \mathrm{IQ}=2,00-3,50$ & \multirow{3}{*}{$H(2)=0,118$} & \multirow{3}{*}{$p=0,943$} \\
\hline & Afinação & 3,$39 ; \mathrm{IQ}=1,69-3,75$ & & \\
\hline & Fundição & 3,$00 ; I Q=2,45-3,57$ & & \\
\hline \multirow{3}{*}{$\begin{array}{l}\text { Sonolência média no } \\
\text { meio do turno }\end{array}$} & Diurno & 3,$50 ; I Q=2,50-5,25$ & \multirow{3}{*}{$H(2)=0,601$} & \multirow{3}{*}{$p=0,741$} \\
\hline & Afinação & 3,$35 ; \mathrm{IQ}=2,31-4,45$ & & \\
\hline & Fundição & 3,$60 ; I Q=3,31-4,75$ & & \\
\hline \multirow{3}{*}{$\begin{array}{l}\text { Sonolência média no } \\
\text { final do turno }\end{array}$} & Diurno & 3,$75 ; I Q=2,50-5,50$ & \multirow{3}{*}{$H(2)=2,636$} & \multirow{3}{*}{$p=0,268$} \\
\hline & Afinação & 4,$40 ; \mathrm{IQ}=3,55-5,00$ & & \\
\hline & Fundição & 4,$54 ; I Q=4,13-5,94$ & & \\
\hline \multirow{3}{*}{$\begin{array}{l}\text { Fadiga média no início } \\
\text { do turno }\end{array}$} & Diurno & 1,$05 ; \mathrm{IQ}=0,38-3,43$ & \multirow{3}{*}{$H(2)=1,205$} & \multirow{3}{*}{$p=0,547$} \\
\hline & Afinação & 1,$82 ; I Q=0,39-3,64$ & & \\
\hline & Fundição & 1,$91 ; I Q=1,35-3,29$ & & \\
\hline \multirow{3}{*}{$\begin{array}{l}\text { Fadiga média no meio } \\
\text { do turno }\end{array}$} & Diurno & 2,$50 ; I Q=1,00-5,00$ & \multirow{3}{*}{$H(2)=2,737$} & \multirow{3}{*}{$p=0,254$} \\
\hline & Afinação & 1,$78 ; \mathrm{IQ}=1,23-2,73$ & & \\
\hline & Fundição & 2,$99 ; I Q=2,69-4,51$ & & \\
\hline \multirow{3}{*}{$\begin{array}{l}\text { Fadiga média no final } \\
\text { do turno }\end{array}$} & Diurno & 3,$00 ; I Q=1,33-5,73$ & \multirow{3}{*}{$H(2)=3,424$} & \multirow{3}{*}{$p=0,180$} \\
\hline & Afinação & 3,$51 ; I Q=2,39-4,55$ & & \\
\hline & Fundição & 4,$19 ; I Q=3,69-6,38$ & & \\
\hline
\end{tabular}

(1) Os resultados da variável correspondem à mediana e ao intervalo interquartil (IQ) entre o primeiro e o terceiro quartil, sendo que a sonolência foi medidas em pontos e a fadiga em centímetros de uma linha de $10 \mathrm{~cm}$.

A maioria dos trabalhadores diurnos registrou sonolência de até 5 pontos em todos os momentos, indicando que se sentiam alertas. Sete trabalhadores apontaram sonolência superior a 5 pontos, normalmente no meio ou no final do turno, e somente um assinalou pontuação superior a 7 ao final do turno (sonolência excessiva). Os trabalhadores noturnos também relataram percepções semelhantes. Quatro deles, dois da afinação e dois da fundição, relataram sonolência acima de 5 pontos no meio ou no final do turno 
de trabalho. Portanto, trabalhadores diurnos e noturnos não se percebiam como sendo muito sonolentos durante o trabalho. Observou-se, porém, que a sonolência aumentou com o transcorrer do turno em todos os grupos, sendo menor entre trabalhadores diurnos, mais evidente entre os trabalhadores noturnos da afinação e mais pronunciada entre os trabalhadores noturnos da fundição. As correlações de Spearman demonstraram que esse aumento foi significativo somente para os trabalhadores noturnos (Figura 1).

Figura 1. Aumento da sonolência ao longo do turno de trabalho

\section{Sonolência}

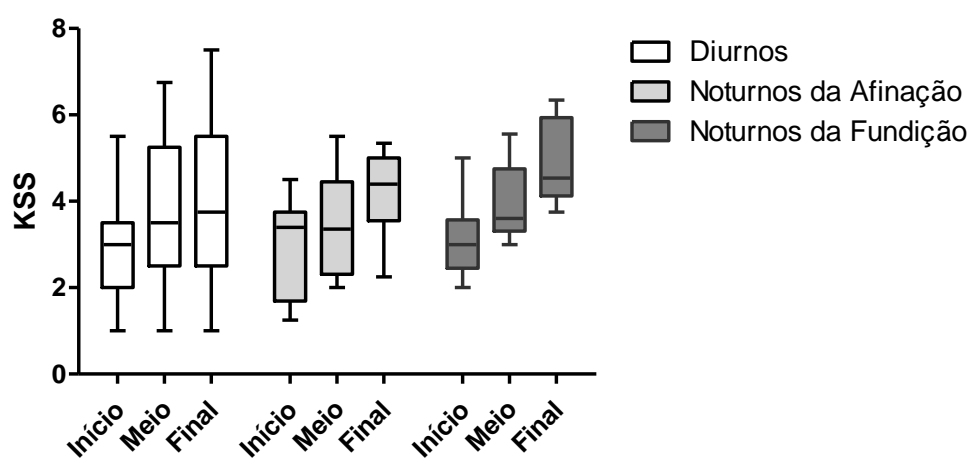

Significativo para os trabalhadores noturnos da afinação ( $r s=0,432, p$ (monocaudal) $<0,019$ ) e da fundição $(\mathrm{rs}=0,659, \mathrm{p}$ (monocaudal) $<0,000)$, mas não para os trabalhadores diurnos $(r s=0,203, p$ (monocaudal $)=$ 0,065).

Sobre a fadiga, os resultados novamente sugerem que a maioria dos trabalhadores estudados não se percebe fatigada ao longo do turno, pois todas as medianas ficaram aquém de $5 \mathrm{~cm}$, ponto intermediário entre nada cansado e cansaço máximo. Sete trabalhadores diurnos relataram fadiga média mais elevada no meio e no final do turno, onde seis foram os mesmos que apontaram sonolências mais intensas. Os valores máximos, de 7,2 e 7,9 cm, foram registrados por dois trabalhadores no final do turno.

Três dos quatro trabalhadores noturnos que relataram mais fadiga também apontaram mais sonolência. Fadigas mais intensas também se concentraram no meio e no final do turno, e o maior valor, de aproximadamente $7,7 \mathrm{~cm}$, foi registrado por um trabalhador da fundição no final da jornada.

Assim, os resultados para a fadiga assemelham-se àqueles obtidos para a sonolência, mas com os valores máximos ao final do turno sendo mais elevados. Além disso, os aumentos na fadiga foram significativos ao longo do turno para todos os trabalhadores, praticamente triplicando entre trabalhadores diurnos e quase dobrando entre os noturnos. Mesmo assim, a percepção de fadiga foi maior entre os trabalhadores noturnos, principalmente os noturnos da fundição (Figura 2). 
Figura 2. Aumento da fadiga ao longo do turno de trabalho

Fadiga

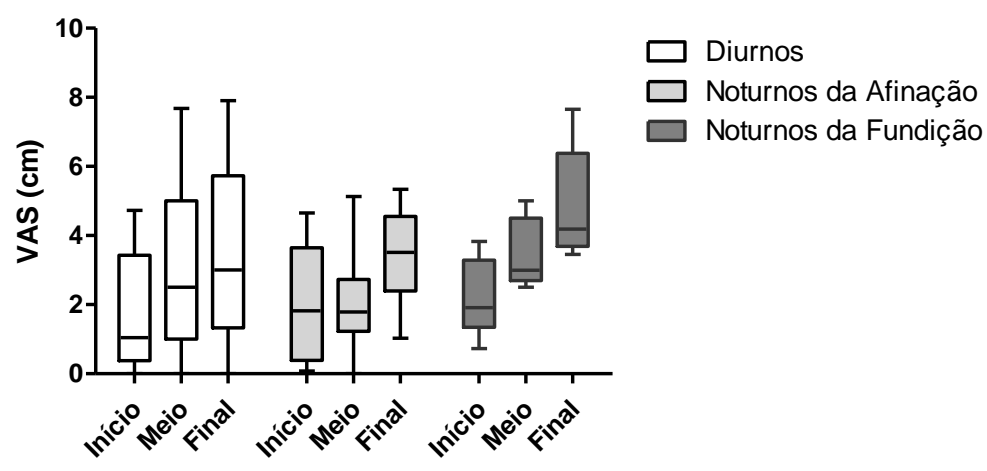

Significativo para os trabalhadores diurnos ( $r s=0,288, p$ (monocaudal) $<0,016$ ), noturnos da afinação ( $r s=$ $0,354, p$ (monocaudal) $<0,046$ ) e noturnos da fundição ( $r s=0,723, p$ (monocaudal) $<0,000$ ), sendo claramente mais pronunciada neste último grupo.

\section{DISCUSSÃo}

\section{Aspectos relacionados ao sono}

O sono diurno dos trabalhadores noturnos deste estudo é compatível com o relatado na literatura, com um tempo na cama de pouco mais de 6 horas e meia (PILCHER; LAMBERT; HUFFCUTT, 2000). Como eles acordavam do sono diurno por volta de 15 horas, esse sono mais curto provavelmente deve-se a um despertar espontâneo (AKERSTEDT, 2003) associado a uma possível dessincronização circadiana crônica típica de trabalhadores noturnos (AKERSTEDT, 2003; DIJK; VON SCHANTZ, 2005; MORRIS; AESCHBACH; SCHEER, 2012).

Em dias de trabalho, as medianas para os horários de dormir e acordar dos trabalhadores diurnos foram, respectivamente, $23 \mathrm{~h} 07 \mathrm{~m}$ e $5 \mathrm{~h} 07 \mathrm{~m}$. Este horário de despertar tão cedo é consequência direta do horário de início do turno de trabalho, às $7 \mathrm{~h}$, tornando-o semelhante a turnos matutinos (SALLINEN; KECKLUND, 2010) e para os quais se observa comumente a necessidade de acordar muito cedo para ir trabalhar (AKERSTEDT, 2003; SALLINEN; KECKLUND, 2010). Assim, o horário de acordar por volta da 5 h, o tempo passado na cama e a duração do sono dos trabalhadores diurnos, em dias de trabalho, assemelham-se mais ao de trabalhadores de turnos matutinos, que têm duração do sono estimada em 6 horas (AKERSTEDT, 2003). Provavelmente também teria havido a interferência do tempo de transporte de casa até o trabalho e o tempo de preparação para a ida ao trabalho. É interessante notar que a dificuldade em acordar muito cedo muitas vezes torna turnos com esta característica bastante impopulares entre os trabalhadores (AKERSTEDT, 2003).

Assim, observou-se que nesta amostra, em dias de trabalho, todos os trabalhadores passavam na cama de 6 a 7 horas e efetivamente dormiam por volta de 5 horas, sendo que o turno de trabalho, diurno ou noturno, não teve papel significativo na duração do sono dos dois grupos.

Conforme as recomendações sobre a duração de sono, adultos na mesma faixa etária dos trabalhadores deste estudo, deveriam dormir diariamente de 7 a 9 horas (HIRSHKOWITZ et al., 2015), quantidade que não foi alcançada por eles em dias de 
trabalho. Essa privação de sono crônica também é sugerida pelo rebote de sono no dia de folga como foi observado. Os trabalhadores noturnos dormiram significativamente mais no dia de folga que os trabalhadores diurnos, sugerindo que seu sono em dias de trabalho é qualitativamente pior, levando-os a uma maior compensação nesse dia (BOIVIN; BOUDREAU, 2014). Dessa forma, a organização do trabalho prejudicou o sono dessa amostra de trabalhadores por motivos distintos: entre os diurnos, o início do turno muito cedo pela manhã acarretou menos tempo passado na cama e uma duração menor do sono; entre os noturnos, a menor duração e uma possível pior qualidade do sono devem-se às mudanças no ciclo vigília-sono decorrentes do trabalho noturno.

\section{Relação entre o sono, a sonolência e a fadiga}

Entre trabalhadores noturnos, o aumento na sonolência estava associado com o transcorrer da jornada de trabalho e foi estatisticamente significativo. Este resultado concorda com resultados anteriores, apesar de os níveis de sonolência relatados terem sido inferiores aos de outros estudos (AKERSTEDT; WRIGHT, 2009; BORGES; FISCHER, 2003; GARDE; HANSEN; HANSEN, 2009).

Entre trabalhadores diurnos, o aumento da sonolência não foi significativo, embora sua jornada diária fosse de 10h, mais longa que as $9 \mathrm{~h}$ dos trabalhadores noturnos. Jornadas de trabalho que se iniciam cedo pela manhã usualmente acarretam maior sonolência no início do turno (AKERSTEDT; WRIGHT, 2009; GARDE; HANSEN; HANSEN, 2009), o que não foi observado neste estudo. No entanto, este aumento estaria mais associado a turnos iniciados até as $6 \mathrm{~h}$ da manhã (AKERSTEDT; WRIGHT, 2009), diferindo do turno diurno estudado, e níveis semelhantes aos aqui observados já foram anteriormente relatados (VANGELOVA, 2008). Acrescenta-se que os níveis de sonolência relatados nos outros momentos da jornada de trabalho e a falta de uma associação significativa do aumento no transcorrer da jornada são compatíveis com o relatado na literatura (AKERSTEDT; WRIGHT, 2009; GARDE; HANSEN; HANSEN, 2009).

Logo, os resultados da sonolência de trabalhadores diurnos e noturnos deste estudo são semelhantes àqueles de pesquisas anteriores. Este aumento foi significativo somente para os trabalhadores noturnos, o que sugere que o principal fator contribuinte foi 0 trabalho em desacordo com a ritmicidade circadiana, geralmente não observada entre os trabalhadores diurnos (AKERSTEDT, 2003).

Comparando-se sonolência e fadiga, observou-se que a fadiga se elevou significativamente para todos os trabalhadores ao longo do turno, enquanto o aumento da sonolência foi significativo apenas para os trabalhadores noturnos. Isso sugere que os trabalhadores as percebiam de forma diferente, confirmando que sonolência e fadiga realmente não se equivalem (ÅHSBERG; GARNBERALE; KJELLBERG, 1997; PHILLIPS, 2015) e em concordância com resultados anteriores (ÅHSBERG; GARNBERALE; KJELLBERG, 1997).

Tendo em vista que a fadiga é causada por esforços físicos, mentais ou ambos (PHILLIPS, 2015), traduzindo-se em percepção de tensão ou exaustão (SHAHID; SHEN; SHAPIRO, 2010), e que os participantes deste estudo desempenhavam principalmente atividades mecânicas, repetitivas e com possíveis graus variados de desgaste, torna-se claro o motivo porque houve um aumento da fadiga ao longo do turno para todos os trabalhadores. Em relação à intensidade, os trabalhadores noturnos relataram mais fadiga ao final do turno que os trabalhadores diurnos, em acordo com 
resultados anteriores (NIU et al., 2011). Segundo Dawson e Fletcher (2001), a fadiga de um turno provavelmente varia em função da duração e do horário desse turno, se diurno ou noturno, sendo influenciada também pelo sistema circadiano. Assim, turnos mais longos respondem por mais fadiga e ela acumula-se mais rapidamente em turnos durante a noite subjetiva que durante o dia subjetivo (DAWSON; FLETCHER, 2001). Logo, pode-se supor que as potenciais alterações circadianas nos trabalhadores noturnos também influenciaram o aumento da fadiga durante a jornada, contribuindo para as diferenças encontradas entre trabalhadores diurnos e noturnos. Mas, não obstante tais resultados, os trabalhadores não relataram níveis excessivos de fadiga diária e nem uma eventual fadiga crônica foi identificada, conforme anteriormente descrito. Dessa forma, apesar de laborarem em atividades relativamente exaustivas, a fadiga resultante aparentemente estaria sendo aliviada por períodos de recuperação após a jornada diária e semanal.

\section{Influência das atividades e condições de trabalho}

Os trabalhadores laboravam em atividades subalternas mecânicas e repetitivas, de baixa exigência cognitiva. As diferenças constatadas nos três grupos influenciaram algumas das variáveis analisadas, como o estresse percebido, a sonolência e a fadiga.

O estresse percebido é influenciado por percepções dos trabalhadores acerca de seu bem-estar e qualidade de vida. Os trabalhadores noturnos da afinação apresentaram os melhores resultados, relatando significativamente menos estresse que os trabalhadores diurnos e noturnos da fundição.

Durante o turno diurno normalmente há mais chefes, mais cobranças e pior relacionamento interpessoal, trazendo prejuízos ao bem-estar de trabalhadores com perfil semelhante aos deste estudo (OLIVEIRA et al., 2006). A empresa estudada encaixava-se nesta descrição e pelo menos alguns trabalhadores estavam cientes dessas diferenças. Assim, embora trabalhadores diurnos e noturnos da afinação tivessem atividades laborais similares em termos de dificuldades e exigências físicas e mentais, provavelmente a maior pressão dos chefes e o potencial pior relacionamento durante o dia foram responsáveis pelos maiores níveis de estresse relatados pelos trabalhadores diurnos.

Entretanto, mesmo com esses elementos menos pronunciados, os trabalhadores noturnos da fundição também apresentaram maiores níveis de percepção de estresse que os trabalhadores noturnos da afinação. Aqui, esse resultado pode ser atribuído às maiores exigências físicas e às piores condições de trabalho a que estavam submetidos os primeiros. Esses fatores também influenciaram, para pior, a percepção de sonolência e fadiga desses trabalhadores.

As variáveis relativas ao sono, entretanto, não foram diferentes quando os trabalhadores noturnos da fundição foram comparados aos da afinação, mas os primeiros apresentaram piores avaliações de aspectos relacionados a seu bem-estar, sonolência e fadiga que se devem provavelmente às piores condições de trabalho a que estavam expostos.

\section{Limitações deste estudo}


O desenho transversal deste estudo não permite estabelecer relações causais entre as variáveis, apenas associações. Também seria recomendável a obtenção de dados durante um intervalo mais prolongado para uma melhor caracterização das associações encontradas.

Outra limitação decorreu do pequeno número de trabalhadores participantes do estudo, talvez contribuindo para que alguns testes estatísticos não tenham mostrado significância estatística. A ausência de sujeitos dos diferentes turnos nos mesmos setores não impediu a obtenção de resultados, mas amostras maiores e mais homogêneas teriam dado mais robustez e confiança às associações estabelecidas.

\section{CONSIDERAÇÕES FinaIS}

A partir do observado neste estudo, demonstra-se que o trabalho noturno e variáveis associadas à realidade de trabalho, como os fatores organizacionais e as próprias condições de trabalho, podem ter efeitos negativos para o bem-estar, o sono, a sonolência e a fadiga dos trabalhadores. Confirmamos que o trabalho noturno prejudica o sono e leva a aumentos mais expressivos da sonolência e da fadiga ao longo do turno de trabalho. Mas, para além do que é normalmente relatado na literatura, também apontamos: a) que sonolência e fadiga são duas variáveis distintas, com causas e consequências diferentes; e b) a contribuição das más condições de trabalho sobre os resultados obtidos neste estudo, levando-nos a concluir que, ao lado dos fatores relacionados à duração e horário da jornada de trabalho, também esses aspectos devem ser considerados ao se realizar a análise de variáveis como sonolência e fadiga.

É interessante explorar um pouco mais esta última questão das condições de trabalho. Há muito tempo é reconhecida a associação entre a extensão da jornada e os horários de trabalho com a perturbação e os prejuízos à saúde e à vida dos trabalhadores, tanto é que a primeira Convenção emitida pela Organização Internacional do Trabalho no ano de sua criação, 1919, aborda justamente as horas de trabalho na indústria (ORGANIZAÇÃO INTERNACIONAL DO TRABALHO, 2015). Esta Convenção não foi ratificada pelo Brasil, mas o país ratificou a Convenção no 171, sobre o trabalho noturno (BRASIL, 2019). Ela trata da proteção daqueles que trabalham ao menos durante parte da noite, sem limitação de gênero ou atividade. Entre outras medidas, ela estabelece os seguintes direitos para os trabalhadores: a) uma avaliação de saúde específica, considerando os efeitos do trabalho noturno, antes e após começar a trabalhar à noite; b) acesso a instalações de primeiros-socorros no ambiente de trabalho; c) transferência, sempre que possível, para uma função similar no turno diurno se o trabalho noturno estiver sendo muito prejudicial à saúde; d) atividades e funções alternativas em outros turnos no caso de gestação; e) compensação financeira especial decorrente do trabalho noturno; e f) oferta de serviços sociais apropriados (BRASIL, 2019).

Esta Convenção também dispõe que os representantes dos trabalhadores deveriam ser consultados, antes da introdução do trabalho noturno e depois regularmente, sobre os detalhes de horários e as formas de organização do trabalho, bem como as medidas de saúde no trabalho e os serviços sociais necessários (BRASIL, 2019).

Verifica-se que, no presente caso, apenas a compensação financeira estava sendo atendida pela empresa. Além disso, as próprias condições de trabalho, durante o dia e à noite, deixaram claro que a empresa não prezava por medidas que tornassem 0 ambiente mais seguro ou saudável. Como a empresa adota turnos semi-contínuos, é questionável se seria necessário haver turnos noturnos, pois os processos de produção 
não exigem continuidade. A implantação do trabalho noturno aparentemente visa apenas 0 aumento da produtividade e lucratividade da empresa, sem considerar os efeitos do trabalho noturno na saúde e na qualidade de vida dos trabalhadores.

Recomenda-se que as avaliações em segurança e saúde do trabalho incluam análises de sono, sonolência e fadiga e de outros fatores relacionados à organização do trabalho.

\section{REFERÊNCIAS}

ÅHSBERG, Elizabeth; GARNBERALE, Francesco; KJELLBERG, Anders. Perceived quality of fatigue during different occupational tasks: Development of a questionnaire. International Journal of Industrial Ergonomics, v. 20, n. 2, p. 121-135, 1997. DOl: 10.1016/S0169-8141(96)00044-3. Disponível em: https://www.sciencedirect.com/science/article/pii/S0169814196000443.

AKERSTEDT, T. Shift work and disturbed sleep/wakefulness. Occupational Medicine, v. 53, n. 2, p. 89-94, 2003. DOI: 10.1093/occmed/kgg046. Disponível em: http://occmed.oupjournals.org/cgi/doi/10.1093loccmed/kgg046.

AKERSTEDT, T.; GILLBERG, M. Sleep disturbances and shiftwork. In: REINBERG, A.; VIEUX, N.; ANDLAUER, P. (org.). Night and Shift Work. Biological and Social Aspects (Advances in the Biosciences 30). Oxford: Pergamon Press, 1981. p. 127-137.

AKERSTEDT, Torbjörn; WRIGHT, Kenneth P. Jr. Sleep loss and fatigue in shift work and shift work disorder. Sleep Medicine Clinics, v. 4, n. 2, p. 257-271, 2009. DOI: 10.1016/j.jsmc.2009.03.001.Sleep. Disponível em: https:/www.sciencedirect.com/science/article/pii/S1556407X09000356.

AMBULATORY MONITORING INC. Motionlogger ${ }^{\circledR}$ User's Guide Version 2K1.1. Ardsley, NY USAAmbulatory Monitoring, Inc., 2000.

ANTUNES, Ricardo. Século XXI: nova era da precarização estrutural do trabalho? São Paulo: Boitempo, 2009.

BERTOLAZI, Alessandra Naimaier; FAGONDES, Simone Chaves; HOFF, Leonardo Santos; PEDRO, Vinícius Dallagasperina; MENNA BARRETO, Sérgio Saldanha; JOHNS, Murray W. Portuguese-language version of the Epworth sleepiness scale: validation for use in Brazil. Jornal Brasileiro de Pneumologia, v. 35, n. 9, p. 877-883, 2009. DOI: 10.1590/S1806-37132009000900009. Disponível em: http://www.scielo.br/scielo.php?script=sci_arttext\&pid=S1806-

37132009000900009\&lng=en\&nrm=iso\&tlng=en.

BOIVIN, Diane B.; BOUDREAU, Philippe. Impacts of shift work on sleep and circadian rhythms. Pathologie Biologie, v. 62, n. 5, p. 292-301, 2014. DOI: 10.1016/.patbio.2014.08.001. Disponível em: http:/www.ncbi.nlm.nih.gov/pubmed/25246026.

BORGES, Flavio Notarnicola da Silva; FISCHER, Frida Marina. Twelve-hour night shifts of healthcare workers: a risk to the patients? Chronobiology International, v. 20, n. 2, p. 351-60, 2003. Disponível em: http:/www.ncbi.nlm.nih.gov/pubmed/12723890.

BORGES, FNDAS. Trabalhadores de enfermagem: compreendendo condições de vida e trabalho e ritmos biológicos. 2006. Universidade de São Paulo, 2006. Disponível em: http://www.teses.usp.br/teses/disponiveis/6/6134/tde-07032007-150549/publico/Borges_FNS.pdf.

BRASIL. Decreto no 10.088, de 5 de novembro de 2019. Consolida atos normativos editados pelo Poder Executivo Federal que dispõem sobre a promulgação de convenções e recomendações da Organização Internacional do Trabalho - OIT ratificadas pela República Federativa do Brasil. Diário Oficial da União, Brasília, 6 de novembro de 2019, Seção 1, Edição 215, páginas 12-135.

DAWSON, Drew; FLETCHER, Adam. A quantitative model of work-related fatigue: background and definition. Ergonomics, v. 44, n. 2, p. 144-63, 2001. Disponível em: http://www.ncbi.nlm.nih.gov/pubmed/11209874.

DIJK, Derk-Jan; VON SCHANTZ, Malcolm. Timing and consolidation of human sleep, wakefulness, and performance by a symphony of oscillators. Journal of Biological Rhythms, v. 20, n. 4, p. 279-90, 2005. DOI: 10.1177/0748730405278292. Disponível em: http:/jbr.sagepub.com/cgi/content/abstract/20/4/279. 
FISCHER, F. M. Método de avaliação do ritmo de trabalho e repouso entre condutores de veículos. In: Primeiro Congresso de Segurança do Tráfego. São Paulo, 1985.

FISCHER, Frida Marina. As demandas da sociedade atual: aspectos históricos do desenvolvimento do trabalho em turnos no mundo - conceitos, escalas de trabalho, legislação brasileira. In: FISCHER, Frida Marina; MORENO, Claudia Roberta De Castro; ROTENBERG, Lúcia (org.). Trabalho em turnos e noturno na sociedade 24 horas. São Paulo: Editora Atheneu, 2004. p. 3-17.

GARDE, Anne Helene; HANSEN, Ase Marie; HANSEN, Johnni. Sleep length and quality, sleepiness and urinary melatonin among healthy Danish nurses with shift work during work and leisure time. International Archives of Occupational and Environmental Health, v. 82, n. 10, p. 1219-28, 2009. DOI: 10.1007/s00420-009-0419-4. Disponível em: http://www.ncbi.nlm.nih.gov/pubmed/19396613.

HIRSHKOWITZ, Max et al. National Sleep Foundation's sleep time duration recommendations: methodology and results summary. Sleep Health, v. 1, n. 1, p. 40-43, 2015. DOI: 10.1016/j.sleh.2014.12.010. Disponível em: http:/www.sleephealthjournal.org/article/S2352721815000157/fulltext.

MORRIS, Christopher J.; AESCHBACH, Daniel; SCHEER, Frank A. J. L. Circadian system, sleep and endocrinology. Molecular and Cellular Endocrinology, v. 349, n. 1, p. 91-104, 2012. DOI: 10.1016/..mce.2011.09.003. Disponível em: http://dx.doi.org/10.1016/j.mce.2011.09.003.

NIU, Shu-Fen; CHUNG, Min-Huey; CHEN, Chiung-Hua; HEGNEY, Desley; O'BRIEN, Anthony; CHOU, Kuei-Ru. The effect of shift rotation on employee cortisol profile, sleep quality, fatigue, and attention level: a systematic review. Journal of Nursing Research, v. 19, n. 1, p. 68-81, 2011. DOI: 10.1097/JNR.0b013e31820c1879. Disponível em: http://www.ncbi.nlm.nih.gov/pubmed/21350389.

OLIVEIRA, Adriana Leônidas De; BERTHOUD, Cristiana Mercadante Esper; BEGLIOMINI, Ângelo Rovaris; COPPOLA, Raquel Gagliotti; RANGEL, Terezinha Chaves. O trabalho noturno e suas repercussões na saúde e na vida cotidiana de trabalhadores metalúrgicos do vale do Paraíba no estado de São Paulo. Revista Psicologia, Organizações e Trabalho, v. 6, n. 2, p. 65-84, 2006. Disponível em: http://pepsic.bvsalud.org/scielo.php?script=sci_arttext\&pid=S1984-66572006000200004.

ORGANIZAÇÃO INTERNACIONAL DO TRABALHO. Hours of Work (Industry) Convention, 1919 (No. 1). In: ORGANIZAÇÃO INTERNACIONAL DO TRABALHO. Compilation of international labour Conventions and Recommendations. Genebra: OIT, 2015. p. 338-342.

PHILLIPS, Ross O. A review of definitions of fatigue - And a step towards a whole definition. Transportation Research Part F: Traffic Psychology and Behaviour, v. 29, p. 48-56, 2015. DOI: 10.1016/j.trf.2015.01.003.

PILCHER, J. J.; LAMBERT, B. J.; HUFFCUTT, A. I. Differential effects of permanent and rotating shifts on self-report sleep length: a meta-analytic review. Sleep, v. 23, n. 2, p. 155-63, 2000. Disponível em: http:/www.ncbi.nlm.nih.gov/pubmed/10737332.

PIRES, M. L. N.; BENEDITO-SILVA, A. A.; MELLO, M. T.; DEL GIGLIO, S.; POMPEIA, C.; TUFIK, S. Sleep habits and complaints of adults in the city of São Paulo, Brazil, in 1987 and 1995. Brazilian Journal of Medical and Biological Research, v. 40, n. 11, p. 1505-1515, 2007. DOI: 10.1590/S0100879X2006005000170. Disponível em: http:/www.scielo.br/scielo.php?script=sci_arttext\&pid=S0100879X2007001100011\&lng=en\&nrm=iso\&tlng=en.

REIS, Rodrigo Siqueira; HINO, Adriano Akira Ferreira; AÑEZ, Ciro Romélio Rodriguez. Perceived stress scale: reliability and validity study in Brazil. Journal of Health Psychology, v. 15, n. 1, p. 107-14, 2010. DOI: 10.1177/1359105309346343. Disponível em: http://www.ncbi.nlm.nih.gov/pubmed/20064889.

SALLINEN, Mikael; KECKLUND, Göran. Shift work, sleep, and sleepiness - differences between shift schedules and systems. Scandinavian Journal of Work, Environment \& Health, v. 36, n. 2, p. 121-33, 2010. Disponível em: http://www.ncbi.nlm.nih.gov/pubmed/20119631.

SHAHID, Azmeh; SHEN, Jianhua; SHAPIRO, Colin M. Measurements of sleepiness and fatigue. Journal of Psychosomatic Research, v. 69, n. 1, p. 81-9, 2010. DOI: 10.1016/j.jpsychores.2010.04.001. Disponível em: http:/www.ncbi.nlm.nih.gov/pubmed/20630266.

SHEN, Jianhua; BARBERA, Joseph; SHAPIRO, Colin M. Distinguishing sleepiness and fatigue: focus on definition and measurement. Sleep Medicine Reviews, v. 10, n. 1, p. 63-76, 2006. DOI: 10.1016/j.smv.2005.05.004. Disponível em: http://www.ncbi.nlm.nih.gov/pubmed/16376590. 
VANGELOVA, Katia. The effect of shift rotation on variations of cortisol, fatigue and sleep in sound engineers. Industrial Health, v. 46, n. 5, p. 490-3, 2008. Disponível em: http://www.ncbi.nlm.nih.gov/pubmed/18840940.

YOSHITAKE, H. Relations between the symptoms and the feeling of fatigue. Ergonomics, v. 14, n. 1, p. 175-86, 1971. DOI: 10.1080/00140137108931236. Disponível em: http://www.ncbi.nlm.nih.gov/pubmed/5117616.

Data da submissão: $24 / 08 / 2020$

Data da aprovação: 11/06/2021 